\title{
Special Lecture
}

\section{An experimental study of muscle function in the lower limb}

\author{
PAUL KLEIN, PhD \\ University of Brussels \\ Brussels, Belgium
}

The simultaneous activity of the rectus femoris and the hamstrings during common movements that occur in walking, in stair climbing, and descending or in cycling has interested many investigators since Lombard's description of this apparent paradox. These muscles have opposite actions at the hip and at the knee. Already, Lombard stated that their action is complementary and not opposite.

In addition to the study of the action of the rectus femoris and hamstrings, this study includes the muscle triceps surae with its two layers: the soleus and the gastrocnemius.

The questions we try to answer are:

1. How is it mechanically possible to explain the complementary activity of the muscle of the thigh?

2 . Is there a restriction in Lombard's paradox, ie, are there one or more conditions to be fulfilled in order to make the muscles complementary?

3 . What is the role of the different layers of the triceps surae during extension of the knee and the ankle?

The methodology adopted is manifold. In vivo, the electromyogram shows the moment of activity of the different muscles. Radiographs were done of the lower limb (pelvis to foot) of a subject standing upright, knees flexed at various degrees, and in positions occurring during stair climbing.

The length of the muscles and their lever arms are determined directly from the radiographs as well as from an articulated model (drawn from the radiograph) permitting the movement in the sagittal plane.

The same parameters are also measured on cadaveric preparations. The pelvis is fixed on a sliding mechanism allowing its rising up, which induces an extension of the hip, knee, and talocrural joints. The muscle length changes are continuously determined. In a second anatomic study, the different lever arms and their variation during movement are established. The technique is based on the tendon excursion method.

Finally, a mathematical model of the lower limb is established, the results of which are in very good agreement with the measurements of the experimental studies.

The length changes of a muscle inform of the action of the muscle when it contracts. Indeed, we adopt the principle that a muscle, when it contracts, can only bring together its points of insertion and cannot separate them. If during a particular movement a muscle is lengthening, then a contraction will induce the opposite movement.

The major results of this study are:

1. In conditions where only the foot is fixed (and not the pelvis nor a part of the body above it), the hamstrings alone cannot extend the knee. Their sole action is the flexion of the knee and the extension of the hip.

2 . Under the same conditions, the rectus femoris will extend the knee and flex the hip. Starting from a position where both the femur and the tibia are inclined with regard to a vertical line, the quadriceps will only align the femur to the tibia. The latter will stay in the same inclined position. As a result, the subject will be unbalanced and will fall in a forward direction.

3 . When both muscle groups are considered together but only the hamstrings will shorten (the rectus femoris acts like a cord), a contraction of the hamstrings will induce an extension of the hip and of the knee. The posterior tilt of the pelvis tightens the rectus femoris. This muscle will extend the knee because the flexor moment of the hamstrings is smaller than the extension moment of the rectus femoris. At the hip joint, the reverse is true: the extension moment of the hamstrings is higher than the flexor moment of the rectus femoris.

It is important to point out that the excursion of the hamstrings is only about $12 \mathrm{~mm}$, which is small with regard to their total length (about 400 $\mathrm{mm})$.

4. The conclusions of point 3 are only true when the subject stands with the ankle, the knee, and the hip flexed and undergoes from this starting position a triple extension.

5. A second restriction to the conclusions of point 3 is yielded by the variation of the lever arms dur- 
ing movement. The complementary action of hamstrings and the rectus femoris is only possible when the knee is flexed less than \pm 55 degrees. Beyond this critical angle, a contraction of the hamstrings will induce an unbalance of the subject posteriorly: the lever arm of the hamstrings is higher than the one of the rectus femoris. This is highlighted by electrostimultion of the hamstrings in vivo for various positions of knee flexion. As long as the subject stands with the knee flexed less than the critical angle, a stimulation of the hamstrings induces a triple extension of the lower limb.

6 . The role of the triceps surae is to extend the talocrural joint, thus righting the tibia and preventing the aforementioned unbalance. The way the triceps performs this movement depends on which part is active. The soleus has to shorten in order to induce an action on the ankle joint. The gastrocnemius has only to behave like a cord. Indeed, when the femur aligns with regard to the inclined tibia, the upper insertion of the gastrocnemius gets farther away, thus lengthening the muscle. When the muscle is inextensible, an extension of the talocrural joint will result, thus righting the tibia.

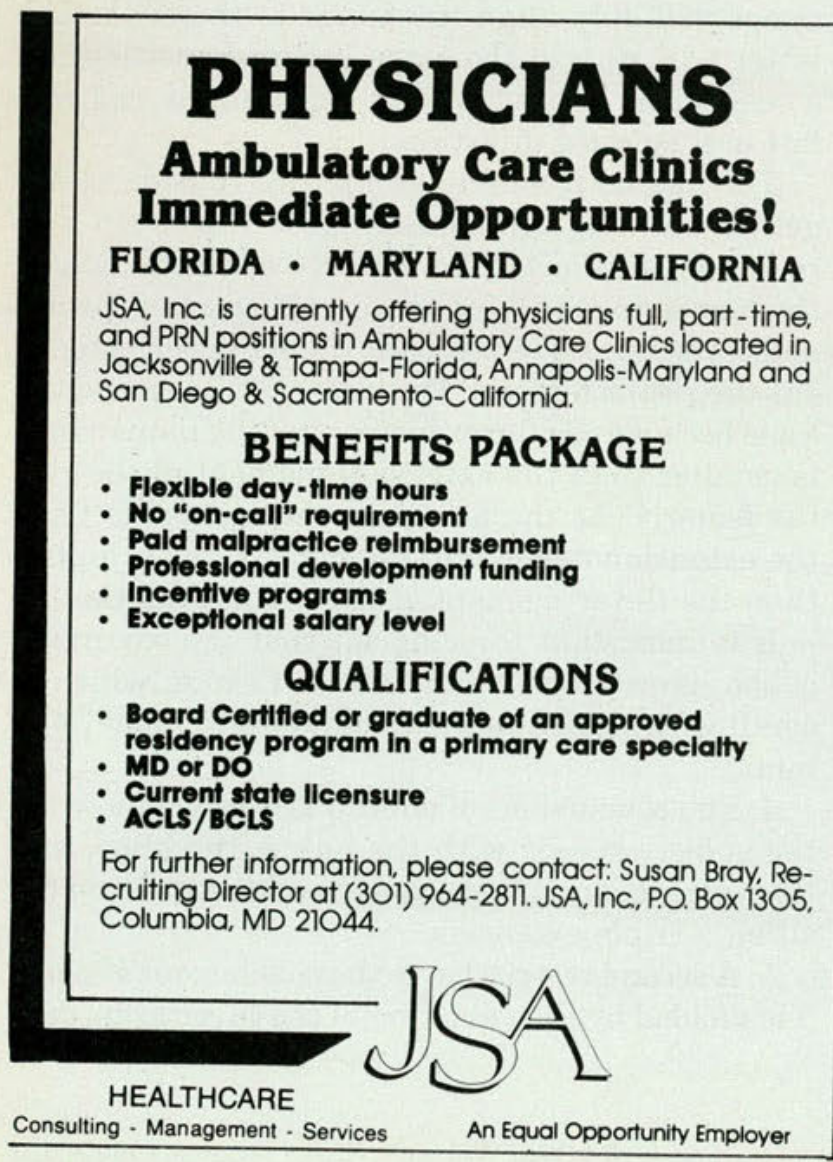

7. Finally, a contraction of the hamstrings induces an extension of the hip joint, an extension of the knee as long as the rectus femoris behaves like a cord, and an extension of the talocrural joint when the gastrocnemius acts also like a cord.

A contraction of the rectus femoris with the two other muscles acting as cords will induce the same result with the difference that the pelvis is much less balanced: the hip is too much flexed with an anterior tilt of the pelvis and a high lumbar lordosis and a subsequent unbalance of the vertebral column.

A shortening of the gastrocnemius will not induce an extension of the knee. The system does not work in this order.

During gait, the hamstrings are in activity just prior to heel strike and during the first part of stance phase. The quadriceps also show an activity. A vector model indicates that these contractions push, respectively pull, the pelvis in a forward direction. This system may represent an energy contribution to progression at the same moment of the push-off of the opposite lower limb. A possible result would be a better equilibration of the pelvis, which is pushed simultaneously from both sides.

In conclusion, even when limited to the sagittal plane, this study emphasizes the role of three muscles acting apparently in an opposite way. By analyzing the variation of their length as well as that of their lever arms, their complementary actions could be pointed out. However, different constraints limit these patterns.

The mechanical relationship between the equilibrium of the pelvis, and consequently that of the vertebral column with the major joints of the lower limb, is shown.

Andrews JG: A general method for determining the functional role of a muscle. J Biomech Eng 1985;107:348-353.

Andrews JG: The functional roles of the hamstrings and quadriceps during cycling: Lombard's Paradox revisited. J Biomech 1987;20(6):565-576.

Elftman H: The function of muscles in locomotion. Am J Physiol 1939;125:357-355.

Gregor RJ, Cavanagh PR, Lafortune M: Knee flexor moments during propulsion in cycling - a creative solution to Lombard's Paradox. J Biomech 1985;18(5):307-316.

Klein P: The knee extension system: Role of the hamstrings. Yearbook of the SSBK 1982;8:31-35 (in French).

Landsmeer JMF: Studies in the anatomy of articulation. Acta Morpho Neerlands Scand, 1960, pp 3-4. 